\title{
Type II Cepheids: evidence for Na-O anticorrelation for BL Her type stars?
}

\author{
V. Kovtyukh, ${ }^{1,2 \star}$ I. Yegorova, ${ }^{3}$ S. Andrievsky,,${ }^{1,2,4}$ S. Korotin, ${ }^{1,5}$ I. Saviane, ${ }^{6}$ \\ B. Lemasle, ${ }^{7}$ F. Chekhonadskikh ${ }^{1,2}$ and S. Belik ${ }^{1,2}$ \\ ${ }^{1}$ Astronomical Observatory, Odessa National University, Shevchenko Park, UA-65014 Odessa, Ukraine \\ ${ }_{2}^{2}$ Isaac Newton Institute of Chile, Odessa branch, Shevchenko Park, UA-65014 Odessa, Ukraine \\ ${ }^{3}$ Universidad Andres Bello, Fernandez Concha 700, Santiago, Chile \\ ${ }^{4}$ GEPI, Observatoire de Paris-Meudon, CNRS, Universite Paris Diderot, F-92125 Meudon Cedex, France \\ ${ }^{5}$ Crimean Astrophysical Observatory, Nauchny UA-298409, Crimea, Ukraine \\ ${ }^{6}$ European Southern Observatory, Alonso de Cordova 3107, Santiago, Chile \\ ${ }^{7}$ Astronomisches Rechen-Institut, Zentrum für Astronomie der Universität Heidelberg, Mönchhofstr. 12-14, D-69120 Heidelberg, Germany
}

Accepted 2018 March 7. Received 2018 February 27; in original form 2017 December 11

\begin{abstract}
The chemical composition of 28 Population II Cepheids and one RR Lyrae variable has been studied using high-resolution spectra. The chemical composition of W Vir variable stars (with periods longer than $8 \mathrm{~d}$ ) is typical for the halo and thick disc stars. However, the chemical composition of BL Her variables (with periods of $0.8-4 \mathrm{~d}$ ) is drastically different, although it does not differ essentially from that of the stars belonging to globular clusters. In particular, the sodium overabundance $([\mathrm{Na} / \mathrm{Fe}] \approx 0.4)$ is reported for most of these stars, and the $\mathrm{Na}-\mathrm{O}$ anticorrelation is also possible. The evolutionary tracks for BL Her variables (with a progenitor mass value of 0.8 solar masses) indicate that mostly helium-overabundant stars $(Y=$ $0.30-0.35$ ) can fall into the instability strip region. We suppose that it is the helium overabundance that accounts not only for the existence of BL Her variable stars but also for the observed abnormalities in the chemical composition of this small group of pulsating variables.
\end{abstract}

Key words: stars: abundances-stars: BL Her type-stars: type II Cepheids-stars: W Vir type.

\section{INTRODUCTION}

Type II Cepheids (here after T2C) are the stars that fall into the instability strip between RR Lyrae and RV Tau stars (Wallerstein 2002; Feast et al. 2008). They are low-mass stars, older and fainter than the classical Cepheids. T2C are divided into sub-classes: the BL Her stars with pulsation period of $1-4 \mathrm{~d}$ and $\mathrm{W}$ Vir stars with pulsation period of 4-20 d (Soszyński et al. 2008). These two sub-classes correspond to the objects at the different evolutionary stages. BL Her stars are currently evolving away from the horizontal branch (HB) towards the asymptotic giant branch (AGB) and can be considered as post-early-AGB stars (Castellani et al. 2007). The W Vir stars cross the instability strip during their blue-loop excursions from the AGB, while they are undergoing helium-shell flashes. RV Tau stars are most likely post-AGB stars with a pulsation period of more than $20 \mathrm{~d}$.

Using a large low-resolution data base, Harris \& Wallerstein (1984) found a large number T2C with $[\mathrm{Fe} / \mathrm{H}]$ between roughly 0 and -1.0 and peculiar velocities $<100 \mathrm{~km} \mathrm{~s}^{-1}$ as well as a wide

^E-mail: vkovtyukh@ukr.net scatter of additional stars with $[\mathrm{Fe} / \mathrm{H}]$ between -1.0 and -2.5 and peculiar velocities $>50 \mathrm{~km} \mathrm{~s}^{-1}$. Hence, the field stars divided themselves into a dominant thick disc population and a rarer group that appeared to be similar to the Cepheids in globular clusters. Cepheids in the Galaxy's nuclear bulge showed metallicities of the thick disc but with velocities up to $250 \mathrm{~km} \mathrm{~s}^{-1}$.

The short period type T2C, often called BL Her stars, show a wide range in $[\mathrm{Fe} / \mathrm{H}]$ (Maas, Giridhar \& Lambert 2007). Many of them like BL Her itself demonstrate $[\mathrm{Fe} / \mathrm{H}]$ value near to zero. However, there are a number of short period $\mathrm{T} 2 \mathrm{C}$ with $[\mathrm{Fe} / \mathrm{H}]$ values in the -1.5 to -2.0 range, which is similar to the metallicity of many globular clusters with short period Cepheids (Clement et al. 2001). We will call the relatively metal-rich stars by their traditional name of BL Her stars and will refer to the metal-poor sub-group as UY Eri stars.

To extend the analysis of metallicities and relative atomic abundances, Maas et al. (2007) analysed spectra of 19 halo and thick disc Cepheids, with periods ranging from 1.1 to $28.6 \mathrm{~d}$ and a range of metallicity from solar to $[\mathrm{Fe} / \mathrm{H}]=-2$ with one exceptional object near -4.0 , which they noted to be similar to post-AGB stars (van Winckel 2003). Maas et al. (2007) paper lists following three significant points: 
1. Among stars with short periods there is a clear separation into two groups. Most BL Her stars have normal metallicities and showed excesses of carbon, nitrogen, and sodium along with thick disc kinematics. The UY Eri type stars are significantly metal-poor and are similar to stars in globular clusters.

2. Stars with periods between 10 and $20 \mathrm{~d}$, such as W Vir itself, show metallicities between about -1.0 and -2.0 and are similar to variables in globular clusters.

3. Stars with periods longer than $20 \mathrm{~d}$ often show refractory and volatile element separation as do RV Tau stars. There are a few stars with periods in the $20-30 \mathrm{~d}$ interval, such as TW Cap, that are as metal-poor as the 10-20 d stars.

In addition, their discussion of individual stars showed the complexity of the relationships of the T2C among each other and as compared with other thick disc and halo populations. Of particular interest is the presence of many BL Her stars with near solar metallicity despite the absence of such stars in globulars like 47 Tuc. Exceptions may be the peculiar clusters NGC 6388 and 6441, which have metallicities similar to that of 47 Tuc and unusually long period RR Lyrae stars. The latter may be related to KP Cyg and UY CrB which Andrievsky et al. (2010) related to the BL Her stars, and the carbon Cepheids V553 Cen and RT TrA (Wallerstein \& Gonzalez 1996; Wallerstein, Matt \& Gonzalez 2000).

In the course of the project realization devoted to the study of the Galactic metallicity gradient using classical Cepheids (VLT observing run 089.D-0489), it appeared that some of the objects turned out to be T2C. In this paper, we have added more T2C previously observed at the Apache Point Observatory to confirm the classification of these objects and to analyse their evolutionary status.

We present spectroscopic analysis of $28 \mathrm{~T} 2 \mathrm{C}$ and one RR Lyraetype star. We determined atmospheric parameters of these stars: $T_{\text {eff }}, \log g, V_{\mathrm{t}}$, and derived LTE and NLTE abundances of the chemical elements. In addition, the description for individual stars and remarks on their classification as T2C are provided.

\section{OBSERVATIONS AND DATA REDUCTION}

Sixteen objects were observed with cross-dispersed Echelle spectrograph UVES at the VLT (observing run 089.D-0489). ${ }^{1}$ The red arm was used, which covers the wavelength region between 4200 and $11000 \AA$. The grating angle was set at $22.668 \mathrm{deg}$, thus allowing to cover a wavelength interval between 4790 and $6810 \AA$. The light is dispersed over two CCD chips, with a gap between 5760 and $5830 \AA$. The slit was set at 1 arcsec width and 12 arcsec length, providing a resolving power of 38700 . The median signal-to-noise ratio $(\mathrm{S} / \mathrm{N})$ per pixel for the upper and lower chip spectra are $\approx 50$ and $\approx 14$, respectively, but with a relatively large range from $\approx 32$ to $\approx 56$ for the upper chip spectrum, and from $\approx 11$ to $\approx 40$ for the lower chip spectrum. The observations were done in service mode; for some objects, several spectra have been obtained. The exposure times were about 20-30 min. The primary data reduction like bias subtraction, flat-field correction, wavelength calibration, sky subtraction, and spectra extraction was done with the UVES pipeline (Ballester et al. 2000).

Twelve objects were observed with the $3.5-\mathrm{m}$ telescope at the Apache Point Observatory, with the ARC Echelle Spectrograph

\footnotetext{
${ }^{1}$ Based on observations collected at the European Organisation for Astronomical Research in the Southern Hemisphere under ESO programme 089.D-0489(A).
}

(ARCES). By using a prism as cross-disperser, the APO Echelle captures the entire spectrum from 3500 to $10400 \AA$. However, the red-sensitive $2048 \times 2048$ chip has decreasing sensitivity for cool stars at the shorter wavelengths and beyond $9000 \AA$. The observations were done as a part of a programme to derive the chemical composition of certain T2C and RR Lyrae stars. These spectra have a resolving power of about 35000 . The exposure times were usually about $10-30 \mathrm{~min}$. The estimated $\mathrm{S} / \mathrm{N}$ at the continuum level depending upon the wavelength interval is about $80-150$. The uncertainty in the determination of velocities is a few tenths of $\mathrm{km} \mathrm{s}^{-1}$.

Spectrum for one object (V553 Cen) was taken from the ESO archive. The star was observed with the Echelle spectrograph HARPS at the ESO La Silla 3.6-m telescope. The spectral range is 4000-6800 $\AA$ with a resolving power of $R=100000$. For BL Her, the additional spectrum was taken from the archive of the Elodie spectrograph at the Observatoire de Haute-Provence 1.93-m telescope $(R=40000,4000-6800 \AA)$.

For some objects (with double lines in the spectrum or with emissions in Fe lines), the chemical composition was not determined. For those objects, the atmospheric parameters are missing in Table 1.

\section{SPECTROSCOPIC ANALYSIS}

In order to normalize the individual spectra to the local continuum level, to identify the lines of different chemical elements, and to measure the equivalent widths (EWs) of the absorption lines, we used the DECH 30 software package. ${ }^{2}$

To determine the effective temperature $T_{\text {eff }}$, we employed the line depth ratios method of Kovtyukh (2007). Having carefully chosen pairs of lines that have a very different dependence on $T_{\text {eff }}$, the ratios of their central depths are entered in polynomial relations previously calibrated. This technique allows the determination of $T_{\text {eff }}$ with a great precision: the use of several tens $(\geq 50)$ of ratios per spectrum leads to uncertainties of the order of $\approx 30-50 \mathrm{~K}$ when $\mathrm{S} / \mathrm{N}>100$ and of $\approx 50-100 \mathrm{~K}$ when $\mathrm{S} / \mathrm{N}<100$. The method is independent of interstellar reddening and only marginally dependent on other characteristics of stars, such as rotation, microturbulence, and metallicity. The surface gravities $\log g$ were computed using the iron ionization balance. The microturbulence velocity $V_{\mathrm{t}}$ was derived considering that the iron abundance obtained from $\mathrm{Fe}_{\mathrm{I}}$ lines are not correlated with the EW of those lines. The adopted value of the metallicity $[\mathrm{Fe} / \mathrm{H}]$ is calculated using the iron abundance obtained from $\mathrm{Fe}_{\mathrm{I}}$ lines. The resulting atmospheric parameters $T_{\mathrm{eff}}$, $\log g$, and $V_{\mathrm{t}}$ are presented in Table 1 .

The abundances of the investigated elements are determined using the LTE and NLTE (nitrogen, oxygen, and sodium) approximations and atmospheric models by Castelli \& Kurucz (2004), computed for the parameters of each star. The solar abundances are computed for the lines from the solar spectrum (Kurucz et al. 1984) with $\log (g f)$ from the VALD data base (Kupka et al. 1999) and the solar model by Castelli \& Kurucz (2004). They are listed in Lemasle et al. (2015).

\subsection{Nitrogen}

Nitrogen abundance was derived for only a few stars using lines at 7442, 7468, 8628, 8680, 8683, 8686, 8703, 8711, 8718, 8728 , when available. Atomic model of this element was described in Lyubimkov et al. (2011).

\footnotetext{
${ }^{2}$ http://www.gazinur.com/DECH-software.html
} 
Table 1. Details of observations and stellar parameters.

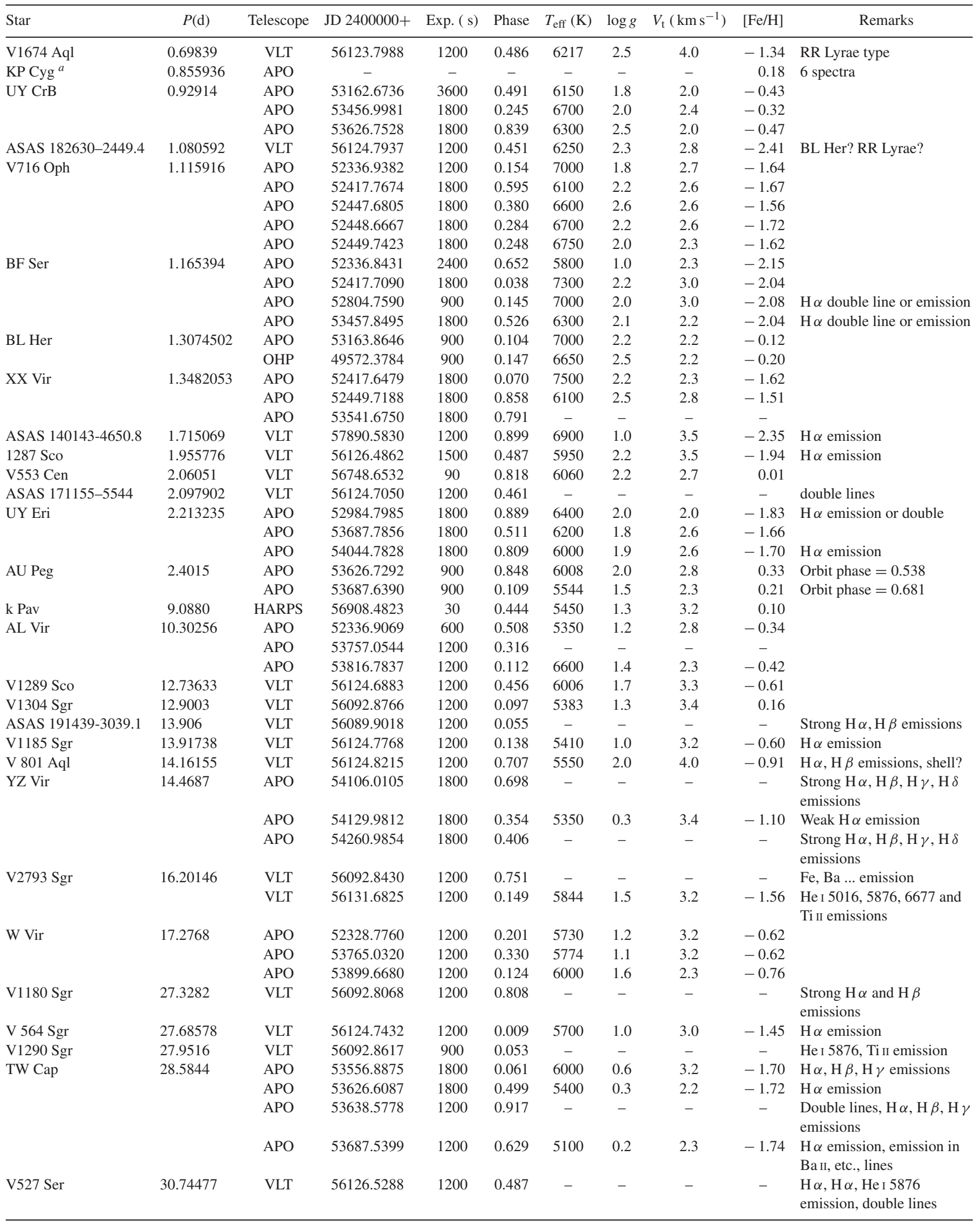


Table 2. Table of $\log (g f)$ and $\chi_{\text {exc }}$ for the sodium and oxygen lines used.

\begin{tabular}{lcr}
\hline Lambda $(\AA)$ & $\chi_{\text {exc }}(\mathrm{eV})$ & $\log (g f)$ \\
\hline & Oxygen & \\
6300.30 & 0.00 & -9.717 \\
7771.94 & 9.11 & 0.369 \\
7774.17 & 9.11 & 0.223 \\
7775.39 & 9.11 & 0.001 \\
8446.25 & 9.52 & -0.462 \\
8446.36 & 9.52 & 0.236 \\
8446.76 & 9.52 & 0.014 \\
& Sodium & \\
6154.23 & 2.09 & -1.56 \\
6160.75 & 2.10 & -1.26 \\
5682.63 & 2.09 & -0.71 \\
5688.19 & 2.10 & -0.41 \\
5889.95 & 0.00 & 0.11 \\
5895.92 & 0.00 & -0.20 \\
\hline
\end{tabular}

\subsection{Oxygen}

The NLTE model of the oxygen atom was first described by Mishenina et al. (2000), and then updated by Korotin et al. (2014). The model consists of $51 \mathrm{O}$ I levels of singlet, triplet, and quintet systems, and the ground level of the $\mathrm{O}_{\text {II }}$ ion. Fine structure splitting was taken into account only for the ground level and the 3p5P level (the upper level of the 7771,2,5 triplet lines). A total of 248 boundbound transitions were included. Oxygen line parameters are listed in Table 2. The high excitation $\mathrm{O}$ I triplet suffers from departure from local thermodynamic equilibrium (Parsons 1964). Parsons showed that its strength depends sensitively on the star's surface gravity. In stars of high luminosity, the triplet is greatly enhanced since radia- tive effects dominate recombination and ionization as compared to collisional excitation which is controlled by the local temperature.

\subsection{Sodium}

We derived the $\mathrm{Na}$ abundances by fitting line profiles (sodium line parameters are given in Table 2). The NLTE atomic model of sodium was presented by Korotin \& Mishenina (1999) and then updated by Dobrovolskas et al. (2005). The updated Na model currently consists of 20 energy levels of $\mathrm{Na}$ I and the ground level of Na II. In total 46 radiative transitions were taken into account for the calculation of the population of all of the levels. For the metal-poor stars, we used the pair at 5682 and $5688 \AA$ and the D lines of Na. For stars with nearly solar metallicity, we used the weaker pair at 6154 and $6160 \AA$. We decided not to use the 8183 and $8194 \AA$ lines because of their blending with absorption by atmospheric lines that depends on the stellar radial velocity and the humidity at the observatory.

Results of the abundance determination in our programme stars are given in Tables 3 and 4.

\section{SOME INDIVIDUAL STARS}

V801 Aql. This star appears in Harris (1985) as T2C, and as pulsating variable in GCVS Kholopov et al. (1985). We note that the spectrum shows strong emission in $\mathrm{H} \alpha$ and $\mathrm{H} \beta$, and also emission in the lines of some ions like $\mathrm{Na}$ I, $\mathrm{Mg}$ I, Y II, and Ba II.

V1674 Aql. There is not too much information about this object in the literature. According to ASAS catalogue this star is of DCEP type with period of $2.3 \mathrm{~d}$. According to GCVS it is RR-Lyraetype star with period of $0.69 \mathrm{~d}$. With a radial velocity of $V_{\mathrm{rad}}=$ $-250 \mathrm{~km} \mathrm{~s}^{-1}$, the RR Lyrae type is a more realistic classification. $\mathrm{H} \alpha$ and $\mathrm{H} \beta$ lines do not show emission.

Table 3. Relative to Fe abundances in $\mathrm{T} 2 \mathrm{C}(\mathrm{C}-\mathrm{Mn})$.

\begin{tabular}{|c|c|c|c|c|c|c|c|c|c|c|c|c|c|c|c|}
\hline Star & $\mathrm{P}$, days & $\mathrm{C}$ & $\mathrm{N}$ & $\mathrm{O}$ & $\mathrm{Na}$ & $\mathrm{Mg}$ & $\mathrm{Al}$ & $\mathrm{Si}$ & S & $\mathrm{Ca}$ & Sc & $\mathrm{Ti}$ & V & $\mathrm{Cr}$ & Mn \\
\hline KP Cyg & 0.855936 & 0.52 & - & 0.14 & 0.30 & -0.04 & 0.20 & -0.02 & 0.10 & -0.04 & -0.03 & 0.06 & 0.02 & -0.12 & -0.03 \\
\hline $\begin{array}{l}\text { ASAS } \\
182630-2449\end{array}$ & 1.080592 & - & - & - & - & 0.52 & - & - & - & 0.49 & 0.27 & 0.37 & - & 0.23 & - \\
\hline V716 Oph & 1.1159157 & 0.43 & - & 0.72 & 0.36 & 0.21 & - & 0.14 & - & 0.53 & 0.23 & 0.49 & 0.64 & 0.15 & 0.64 \\
\hline XX Vir & 1.348205 & - & - & 0.83 & -0.35 & 0.19 & - & 0.05 & - & 0.46 & 0.26 & 0.39 & - & 0.01 & 0.45 \\
\hline $\begin{array}{l}\text { ASAS } \\
140143- \\
4650.8\end{array}$ & 1.715069 & - & - & 0.85 & 0.14 & 0.60 & - & 0.79 & - & 0.56 & 0.04 & 0.23 & - & 0.39 & - \\
\hline V1287 Sco & 1.955776 & - & - & - & - & 0.55 & - & - & - & 0.52 & 0.25 & 0.47 & - & 0.24 & - \\
\hline V553 Cen & 2.06051 & 0.78 & - & -0.11 & 0.43 & 0.19 & 0.12 & 0.00 & 0.10 & 0.23 & 0.28 & 0.28 & 0.01 & 0.01 & -0.02 \\
\hline V1289 Sco & 12.73633 & -0.18 & - & 0.66 & -0.07 & 0.21 & 0.53 & 0.28 & 0.08 & 0.27 & 0.31 & 0.43 & 0.72 & -0.17 & -0.22 \\
\hline V1304 Sgr & 12.9003 & 0.20 & - & 0.12 & 0.04 & -0.16 & 0.20 & -0.02 & 0.14 & 0.05 & 0.02 & 0.21 & -0.05 & -0.18 & -0.10 \\
\hline V1185 Sgr & 13.91738 & -0.10 & - & 0.72 & 0.02 & 0.13 & -0.02 & 0.22 & 0.23 & 0.00 & -0.34 & 0.14 & -0.06 & -0.23 & -0.36 \\
\hline V801 Aql & 14.16155 & 0.58 & - & - & 0.01 & 0.45 & 0.43 & 0.31 & 0.43 & 0.29 & 0.18 & 0.47 & 0.30 & 0.02 & -0.23 \\
\hline YZ Vir & 14.471887 & -0.28 & -0.14 & 0.58 & 0.19 & 0.11 & -0.08 & 0.28 & 0.38 & 0.13 & -0.48 & 0.14 & -0.16 & -0.27 & -0.39 \\
\hline V2793 Sgr & 16.20146 & - & - & - & - & 0.51 & - & 0.37 & 0.49 & 0.32 & -0.07 & 0.39 & - & -0.09 & -0.11 \\
\hline W Vir & 17.2736 & -0.32 & - & 0.74 & 0.13 & 0.28 & 0.16 & 0.22 & 0.15 & -0.01 & -0.94 & 0.21 & 0.02 & -0.25 & -0.27 \\
\hline V564 Sgr & 27.68578 & - & - & - & - & 0.39 & - & 0.40 & - & 0.35 & -0.07 & 0.04 & - & -0.06 & -0.23 \\
\hline TW Cap & 28.5844 & -0.37 & - & 0.97 & - & 0.28 & - & - & 0.66 & 0.34 & -0.01 & 0.30 & 0.40 & -0.11 & 0.05 \\
\hline
\end{tabular}


Table 4. Relative to $\mathrm{Fe}$ abundances in $\mathrm{T} 2 \mathrm{C}(\mathrm{Co}-\mathrm{Eu})$.

\begin{tabular}{|c|c|c|c|c|c|c|c|c|c|c|c|c|c|c|}
\hline Star & $\mathrm{P}$, days & {$[\mathrm{Fe} / \mathrm{H}]$} & Co & $\mathrm{Ni}$ & $\mathrm{Cu}$ & $\mathrm{Zn}$ & $\mathrm{Y}$ & $\mathrm{Zr}$ & $\mathrm{Ba}$ & $\mathrm{La}$ & $\mathrm{Ce}$ & $\mathrm{Nd}$ & $\mathrm{Sm}$ & Eu \\
\hline V1674 Aql & 0.69839 & -1.35 & - & 0.04 & - & 0.26 & -0.01 & - & 0.15 & - & - & 0.45 & - & 0.63 \\
\hline KP Cyg & 0.855936 & 0.18 & -0.10 & 0.00 & -0.37 & 0.38 & -0.04 & -0.04 & -0.14 & 0.12 & -0.08 & -0.02 & - & 0.17 \\
\hline $\mathrm{UY} \mathrm{CrB}$ & 0.92914 & -0.40 & 0.27 & 0.02 & -0.28 & 0.47 & -0.13 & 0.01 & -0.07 & -0.25 & -0.08 & -0.08 & - & 0.57 \\
\hline ASAS 182630-2449 & 1.080592 & -2.41 & - & 0.29 & - & - & 0.36 & - & -0.23 & - & - & - & - & - \\
\hline V716 Oph & 1.1159157 & -1.64 & - & 0.32 & 0.07 & 0.30 & 0.18 & - & -0.05 & - & - & - & - & - \\
\hline BF Ser & 1.165394 & -2.08 & - & - & - & - & 0.28 & - & 0.20 & - & - & - & - & 0.74 \\
\hline BL Her & 1.3074502 & -0.16 & 0.24 & 0.08 & -0.05 & 0.05 & -0.01 & -0.13 & 0.01 & 0.03 & -0.04 & 0.32 & - & 0.38 \\
\hline XX Vir & 1.348205 & -1.55 & - & 0.34 & - & - & 0.10 & - & -0.08 & - & - & - & - & - \\
\hline ASAS 140143-4650.8 & 1.715069 & -2.35 & - & - & - & - & - & - & -0.24 & - & - & - & - & - \\
\hline V1287 Sco & 1.955776 & -1.94 & - & 0.18 & - & 0.37 & 0.15 & - & 0.16 & - & - & - & - & - \\
\hline V553 Cen & 2.06051 & 0.01 & 0.14 & -0.01 & 0.18 & 0.14 & 0.00 & - & - & -0.06 & -0.16 & -0.11 & - & -0.04 \\
\hline UY Eri & 2.213235 & -1.73 & - & 0.34 & 0.17 & 0.31 & 0.06 & - & -0.47 & - & - & - & - & - \\
\hline AU Peg & 2.4015 & 0.26 & -0.09 & -0.03 & 0.18 & 0.15 & -0.32 & -0.21 & -0.12 & -0.33 & -0.30 & -0.20 & - & 0.04 \\
\hline k Pav & 9.0880 & 0.10 & 0.01 & -0.02 & 0.01 & 0.12 & -0.41 & -0.40 & - & -0.54 & -0.46 & -0.64 & - & -0.14 \\
\hline AL Vir & 10.30256 & -0.37 & 0.13 & 0.05 & -0.08 & 0.13 & -0.62 & -0.45 & -0.21 & -0.48 & -0.50 & -0.51 & - & 0.04 \\
\hline V1289 Sco & 12.73633 & -0.61 & 0.10 & 0.09 & -0.06 & 0.28 & 0.45 & - & - & 0.52 & 0.25 & 0.50 & - & 0.64 \\
\hline V1304 Sgr & 12.9003 & 0.16 & 0.06 & 0.03 & -0.02 & - & -0.06 & 0.16 & - & 0.10 & -0.17 & -0.06 & 0.49 & 0.39 \\
\hline V1185 Sgr & 13.91738 & -0.60 & 0.19 & 0.04 & -0.05 & 0.32 & -0.49 & -0.13 & - & -0.49 & -0.35 & -0.52 & -0.10 & 0.09 \\
\hline V801 Aql & 14.16155 & -0.91 & 0.11 & -0.01 & 0.04 & 0.44 & 0.84 & - & - & 0.38 & 0.53 & 0.53 & - & 0.53 \\
\hline YZ Vir & 14.471887 & -1.10 & 0.15 & -0.06 & -0.19 & -0.04 & -0.70 & -0.21 & - & -0.17 & -0.35 & -0.19 & -0.15 & 0.37 \\
\hline V2793 Sgr & 16.20146 & -1.56 & - & 0.09 & - & 0.31 & 0.00 & 0.36 & 0.20 & - & -0.04 & 0.04 & - & 0.51 \\
\hline W Vir & 17.2736 & -0.65 & 0.25 & 0.03 & 0.12 & 0.47 & -0.77 & -0.30 & -0.04 & -0.05 & -0.13 & -0.10 & - & 0.33 \\
\hline V564 Sgr & 27.68578 & -1.45 & - & 0.07 & - & 0.32 & -0.04 & 0.24 & 0.56 & - & - & 0.24 & 0.25 & 0.54 \\
\hline TW Cap & 28.5844 & -1.72 & - & -0.10 & - & 0.28 & -0.03 & - & 0.09 & 0.42 & 0.01 & 0.23 & - & 0.22 \\
\hline
\end{tabular}

TW Cap. The obtained metallicity $[\mathrm{Fe} / \mathrm{H}]=-1.7$ is in agreement with the previous results of Pancino et al. (2015) and Maas et al. (2007). $\mathrm{H} \alpha$ line shows emission components. With a pulsation period of $29 \mathrm{~d}$, TW Cap falls into RV-Tau-type star range (the postAGB phase).

$A U$ Peg. AU Peg is a spectroscopic binary star (Harris, Olszewski \& Wallerstein 1984) with an orbital period of $53.3 \mathrm{~d}$. The chemical composition of this star was studied by Harris et al. (1984) and Maas et al. (2007). Because of the unusual chemical composition, AU Peg cannot be considered as a typical BL Her type.

V564 Sgr. Harris (1985) classified this star as probable T2C with a period of $27.9 \mathrm{~d}$. From its metallicity, one can conclude that most probably it is RV-Tau-type star. We find that spectrum shows strong $\mathrm{H} \alpha$ and $\mathrm{H} \beta$ emissions.

V1180 Sgr. A star of RV Tau type according to SIMBAD and W Vir type according to GCVS and Harris (1985). In the spectrum, strong $\mathrm{H} \alpha$ and $\mathrm{H} \beta$ emissions are present.

V1185 Sgr. W Vir type star according to SIMBAD. H $\alpha$ emission is seen. Fe I lines show redshifted weak emissions.

$V 1290 \mathrm{Sgr}$. $\mathrm{H} \alpha$ and $\mathrm{H} \beta$ show strong emission. He I 5016, 5876, $6677 \AA$ Aines show strong emission, while all other lines are double, and $\mathrm{Ti}$ II lines show blueshifted emission (the star is near maximum light).

V1304 Sgr. W Vir type. $\mathrm{H} \alpha$ and $\mathrm{H} \beta$ are double.

V2793 Sgr. In Harris (1985), this star appears as a T2C. $\mathrm{H} \alpha$ and $\mathrm{H} \beta$ show emission. He I 5016, 5876, $6677 \AA$ lines show emission. Ti II lines show emission as well.

V1287 Sco. Variable star of W Vir type. There is not much information in the literature about this star. $\mathrm{H} \alpha$ emission is seen in the spectrum. The lines are slightly asymmetric.

V1289 Sco. W Vir type according to SIMBAD. CWA with $\mathrm{P}$ $=12.736 \mathrm{~d}$ according to GCVS, or DCEP with $\mathrm{P}=12.73633 \mathrm{~d}$ according to ASAS. $\mathrm{H} \alpha$ line shows weak redshifted emission. At the same time, $\mathrm{H} \beta$ line does not show emission.
BF Ser. All four spectra were taken at important phases. This star is very likely a member of the globular cluster-like group. It is a metal-poor star with the pulsation period of $1.2 \mathrm{~d}$ of the BL Her type. Drake et al. (2014) classified it as BL Boo type star.

V527 Ser. W Vir type according to SIMBAD. He I 5016, 5876, $6677 \AA$ lines show emission. $\mathrm{H} \alpha$ and $\mathrm{H} \beta$ also show emission.

W Vir. A prototype star that has been well studied (see e.g. Kovtyukh et al. 2011 and references therein). We found $[\mathrm{Fe} / \mathrm{H}]$ of -0.70 , while Maas et al. (2007) found -0.95 . This is a reasonable agreement for a variable star.

$X X$ Vir. An interesting object with a short pulsation period of a BL Her type star $(1.3 \mathrm{~d})$, but with a metallicity near $[\mathrm{Fe} / \mathrm{H}]=-1.6$ that places this star in the globular cluster-like group and W Vir type. According to Drake et al. (2014), it is an anomalous Cepheid of BL Boo type.

YZ Vir. This star was not well studied before. Its $[\mathrm{Fe} / \mathrm{H}]$ value falls between metallicities of the BL Her class and the globular cluster class. However, its period places is exactly in the globular cluster class, like W Vir itself.

$A L$ Vir. A metallicity of $[\mathrm{Fe} / \mathrm{H}]=-0.37$ places this star in the BL Her class, but its period of $10 \mathrm{~d}$ does not fit this classification. The metallicity is compatible with the previous value $[\mathrm{Fe} / \mathrm{H}]=$ 0.1 (Schmidt, Rogalla \& Thacker-Lynn 2011) and $[\mathrm{Fe} / \mathrm{H}]=-0.4$ by Maas et al. (2007). Schmidt et al. (2004) noticed He I $5876 \AA$ emission.

ASAS 171155-5544. In ASAS catalogue, this star listed as classical Cepheid type object. Spectrum shows double lines. Narrow spectral lines overlap with the broad-redshifted lines. This may be a binary system.

ASAS 182630-2449. The star of classical Cepheid type according to ASAS catalogue with $\mathrm{P}=1.080592 \mathrm{~d}$. It has a metallicity of $[\mathrm{Fe} / \mathrm{H}]=-2.5$. Taking into account the short period of this star, it can be classified as of BL Her or RR Lyrae type. Spectrum shows asymmetric lines. $\mathrm{H} \alpha$ and $\mathrm{H} \beta$ are asymmetric without emission. 
ASAS 191439-3039. $\mathrm{H} \alpha$ and $\mathrm{H} \beta$ show very strong emission. Spectrum shows double lines.

\section{DISCUSSION}

The T2C stars may be divided into $\mathrm{W}$ Vir stars with periods from 8 to $30 \mathrm{~d}$ (though stars with periods greater than $20 \mathrm{~d}$ often show mild RV Tau properties) and BL Her stars with periods less than $4 \mathrm{~d}$. One of the conclusion of this paper consists in the separation of the 1-4 d stars into the BL Her group with near-solar metallicity and the distinctly metal-poor group that we call the UY Eri class. The latter are to be found also in metal-poor globular clusters (Clement et al. 2001). The relatively metal-rich globulars with $[\mathrm{Fe} / \mathrm{H}]>-1.0$ do not have Cepheids with periods less than $4 \mathrm{~d}$. Of 13 stars in Table 1 of this paper, 7 fall into the metal-poor UY Eri group. All stars of this group show $[\mathrm{Fe} / \mathrm{H}]<-1.5$. Of the eight stars in the Maas et al. (2007) paper, only one star, UY Eri itself, shows $[\mathrm{Fe} / \mathrm{H}]<-1.5$. Three stars appear in both papers.

We wait for the discovery and observation of additional T2C stars by Gaia and eventually LSST. The Gaia spectra in the 8400-8800 region may be useable to derive metallicities from the strength of the Ca II IR triplet that was calibrated for RR Lyrae stars by Wallerstein, Gomez \& Huang (2012).

\subsection{Sodium versus oxygen}

In this section, we will mostly discuss NLTE abundances of oxygen and sodium in aspect of $\mathrm{Na}-\mathrm{O}$ anticorrelation. Population II pulsating variable stars (RR Lyrae, BL Her, and W Vir types) are located in the same instability strip. According to the GCVS catalogue, there are about 8400 RR Lyrae variables (with periods in the range $P=0.2-1.2 \mathrm{~d})$, only $101 \mathrm{BL}$ Her variables $(P=0.8-8 \mathrm{~d})$, and $116 \mathrm{~W}$ Vir variables $(P=8-35 \mathrm{~d})$. A very small number of BL Her variables (whose masses only slightly exceed that of the RR Lyraes) is indicative of the uniqueness of this stage of stellar evolution.

Regarding their masses and metallicities, BL Her type stars are similar to the globular cluster giants. There are two types of models that describe the evolution of globular cluster stars. Langer, Hoffman \& Sneden (1993) describes how low- to medium-mass star internal nuclear reactions could deplete oxygen and enhance sodium by proton captures deep in their interiors, with their products then being convected to the surface.

Two reactions:

${ }^{16} \mathrm{O}(p, \gamma){ }^{17} F(\beta, v){ }^{17} \mathrm{O}(p, \alpha){ }^{14} \mathrm{~N}$

and

${ }^{22} \mathrm{Ne}(p, \gamma){ }^{23} \mathrm{Na}$

lead to an exhaustion of the ${ }^{16} \mathrm{O}$ nuclei and an enhancement of ${ }^{23} \mathrm{Na}$ nuclei in a reaction zone. The first reaction converts ${ }^{16} \mathrm{O}$ into fluorine nuclei that $\beta$ decay to ${ }^{17} \mathrm{O}$ isotope. After proton capture, the stable nuclei of nitrogen are formed. Characteristic time of the following proton capture by ${ }^{14} \mathrm{~N}$ is longer than expected dredge-up time; therefore, material from reaction zone enriched in nitrogen and depleted in oxygen may appear at the star surface altering its superficial composition. The same apply for sodium which is also enhanced at the stellar surface. This mechanism is likely responsible for the surface abundance anomalies in the globular cluster giants that show oxygen deficiency accompanied by sodium excess as reported e.g. by Carretta et al. (2009). Since oxygen is depleted, the nitrogen should be overabundant. Thus, globular cluster giants should show the $\mathrm{Na}-\mathrm{N}$ correlations and $\mathrm{Na}-\mathrm{O}$ anticorrelation

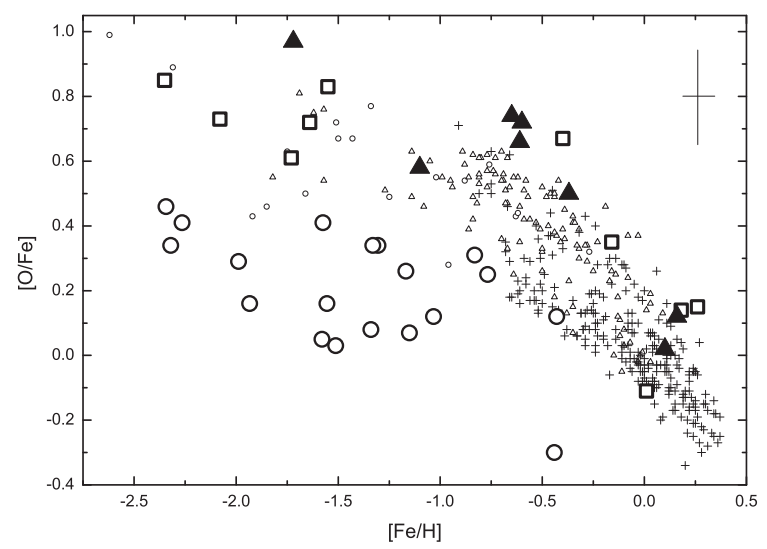

Figure 1. $[\mathrm{O} / \mathrm{Fe}]$ versus $[\mathrm{Fe} / \mathrm{H}]$. Big open squares - BL Her type, filled big triangles - W Vir type, open big circles - globular clusters (Carretta et al. 2009), open small circles - halo stars, open small triangles - thick disc stars, crosses - thin disk stars (Bensby, Feltzing \& Oey 2014). Typical errors are shown.

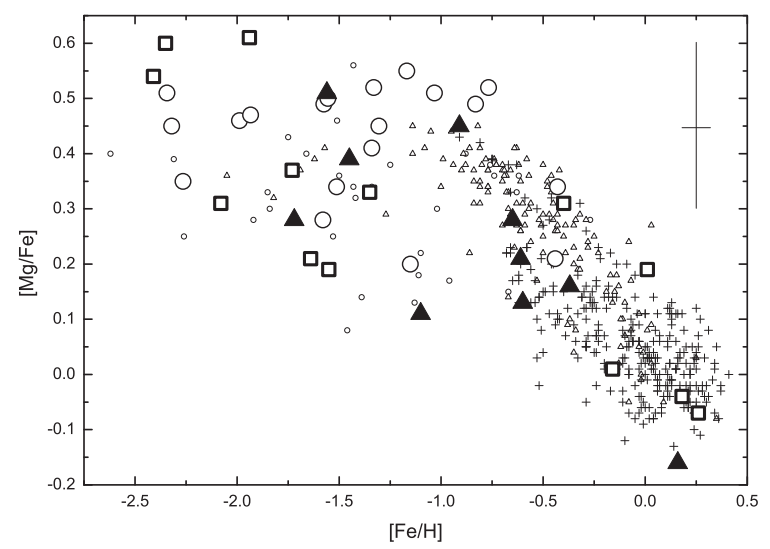

Figure 2. $[\mathrm{Mg} / \mathrm{Fe}]$ versus $[\mathrm{Fe} / \mathrm{H}]$. Designations are the same as in Fig. 1.

(Langer et al. 1993). In addition, an excess of $\mathrm{Al}$ and depletion of $\mathrm{Mg}$ in globular clusters is predicted and often observed.

An entirely different scenario requires at least two generations of stars, the first of which includes some massive, rapidly rotating stars with deep mixing so that the products of nuclear reactions are brought to the surface and swept by stellar wind into the clusters interstellar medium, where they can participate in the formation of subsequent star generation. This model has been reviewed by Charbonnel (2016), see also Renzini et al. (2015).

From Fig. 1, one can see that oxygen abundance in BL Her stars is similar to what is seen in W Vir stars, and both these types follow the oxygen versus iron abundance typical for thick disc and halo field stars. This is also valid for $\alpha$-process element magnesium (Fig. 2) and mean abundance of four $\alpha$-elements $\mathrm{Mg}, \mathrm{Si}, \mathrm{Ca}$, and Ti (Fig. 3). Globular cluster giants show significantly lower oxygen abundance (in Fig. 1, we show the mean oxygen abundance for each cluster).

We succeeded to measure nitrogen abundance only in four stars. The results are controversial. Two stars are nitrogen rich, while other two have normal nitrogen abundance. Among two nitrogenrich stars, one is BL Her itself with a not very high oxygen overabundance, and probably UY CrB can be classified as of BL Her type.

Sodium abundance in most BL Her stars is close to that of globular cluster stars, and both these groups show much higher abundance than disc and halo (including W Vir) stars do (Fig. 4, see also Maas 


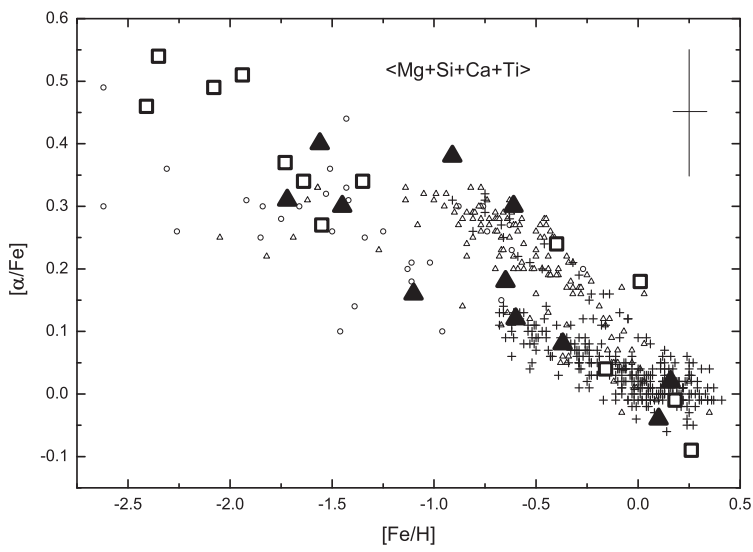

Figure 3. $[\alpha / \mathrm{Fe}]$ versus $[\mathrm{Fe} / \mathrm{H}]$. Designations are the same as in Fig. 1.

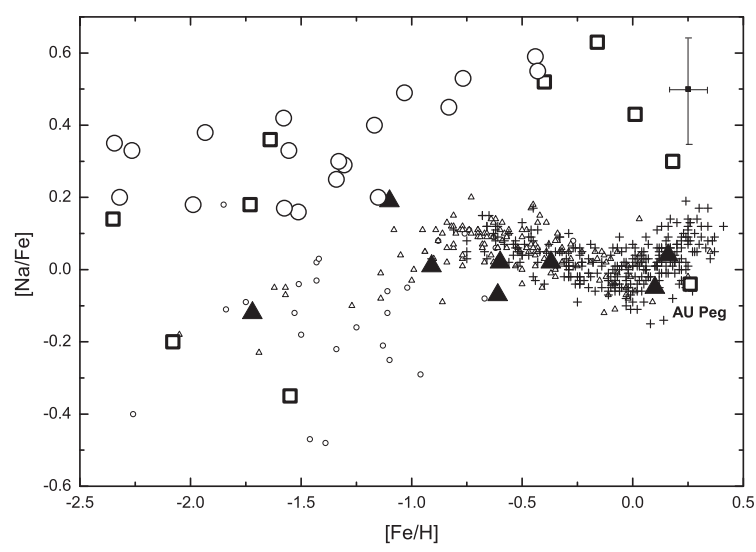

Figure 4. $[\mathrm{Na} / \mathrm{Fe}]$ versus $[\mathrm{Fe} / \mathrm{H}]$. Designations are the same as in Fig. 1.

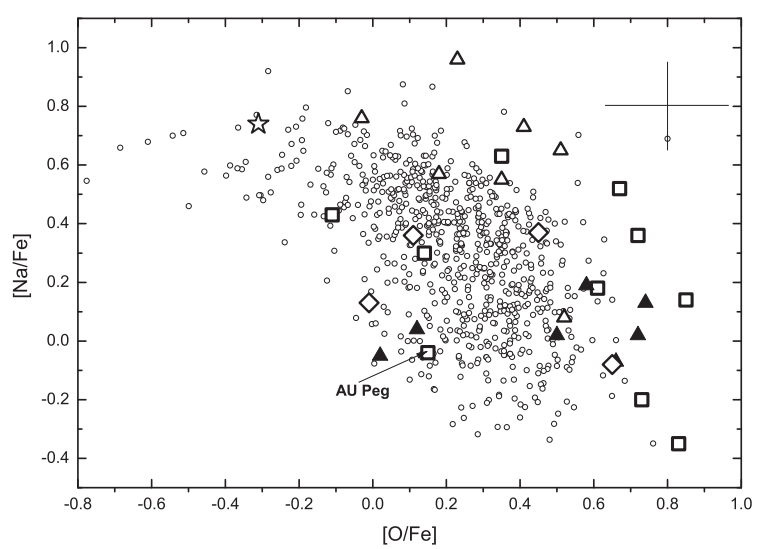

Figure 5. Possible $[\mathrm{Na} / \mathrm{Fe}]$ versus $[\mathrm{O} / \mathrm{Fe}]$ anticorrelation for BL Her type stars. Big open squares - BL Her type, filled big triangles - W Vir type, open small circles - globular clusters giants (Carretta et al. 2009), open big triangles - BL Her stars from Maas et al. (2007), open asterisk - RT TrA (BL Her type star, Wallerstein et al. 2000), open diamonds - V526 Aql, HK Cas, EK Del and GP Per (BL Her type stars, Luck \& Lambert 2011).

et al. 2007; Lemasle et al. 2015). Only BF Ser and XX Vir show $[\mathrm{Na} / \mathrm{Fe}]<0$.

Fig. 5 shows the variation of $[\mathrm{Na} / \mathrm{Fe}]$ as a function of $[\mathrm{O} / \mathrm{Fe}]$. Our BL Her programme stars, combined with the data of Maas et al. (2007), Wallerstein et al. (2000), and Luck \& Lambert (2011), show a clear trend for sodium to increase when oxygen decreases. $\mathrm{BL}$ Her stars seem to follow the $\mathrm{Na}-\mathrm{O}$ anticorrelation observed

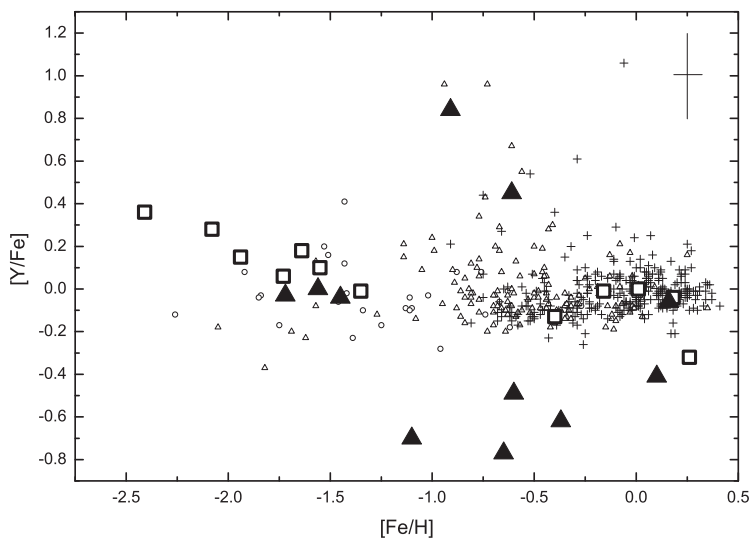

Figure 6. $[\mathrm{Y} / \mathrm{Fe}]$ versus $[\mathrm{Fe} / \mathrm{H}]$. Designations are the same as in Fig. 1 Stars with $[\mathrm{Y} / \mathrm{Fe}]<-0.2$ show the signature of severe dust-gas separation.

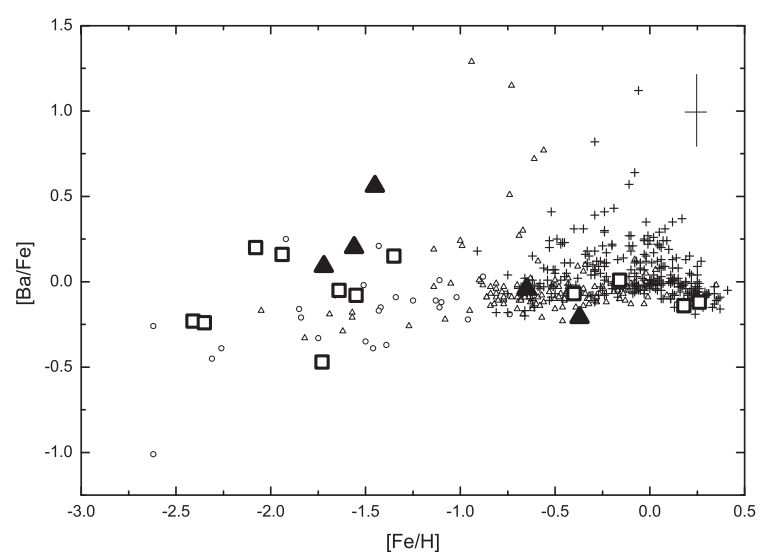

Figure 7. $[\mathrm{Ba} / \mathrm{Fe}]$ versus $[\mathrm{Fe} / \mathrm{H}]$. Designations are the same as in Fig. 1.

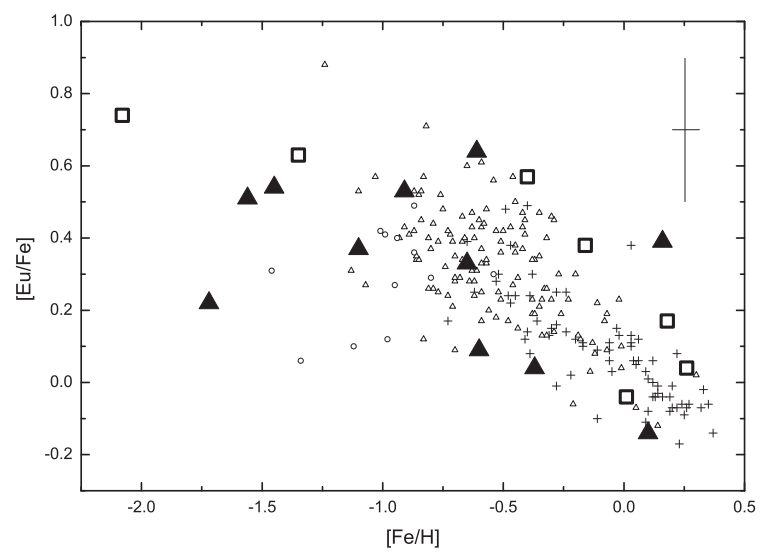

Figure 8. $[\mathrm{Eu} / \mathrm{Fe}]$ versus $[\mathrm{Fe} / \mathrm{H}]$. Big open squares - BL Her type, filled big triangles - W Vir type, open small circles - halo stars (Reddy et al. 2006), open triangles - thick disc stars (Bensby et al. 2005; Reddy et al. 2006), crosses - thin disc stars (Bensby et al. 2005; Reddy et al. 2006).

in globular cluster stars. In contrast, W Vir stars do not show any sodium overabundance or $\mathrm{Na}-\mathrm{O}$ anticorrelation.

Finally, one should note that s- and r-process element distribution with iron contents shows that BL Her behave similarly to the stars from thick disc and halo field. It is demonstrated by Figs 6, 7, and 8 for yttrium, barium, and europium, respectively. The spread in abundances of $\mathrm{Y}, \mathrm{Ba}$ at around $[\mathrm{Fe} / \mathrm{H}] \approx-1$ is the main indicator of 


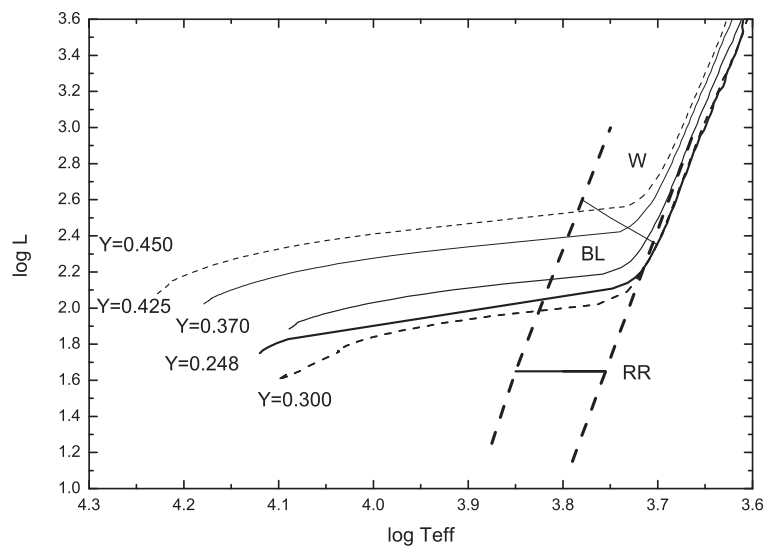

Figure 9. Evolutionary tracks for star with a mass $0.8 \mathrm{M}_{\odot}$ and $[\mathrm{Fe} / \mathrm{H}]=$ -1.75 for various helium abundance $\mathrm{Y}=0.248,0.30,0.37,0.425,0.45$ according to Chantereau, Charbonnel \& Meynet (2016). For clarity reasons, shown only the fraction of the evolutionary tracks covering the post-HB evolution of the stars.

the contribution of the spinstars (Chiappini et al. 2011a,b; Chiappini 2013).

\section{WHAT IS THE ORIGIN OF BL HER STARS?}

As we know, a spread in helium abundance would be expected in second-generation globular cluster stars if the changes in $\mathrm{O}, \mathrm{Na}$, $\mathrm{Mg}$, and $\mathrm{Al}$ are due to proton captures in a previous generation stars in a globular cluster. The multiple main sequences seen in Omega Cen and other clusters such as NGC 2808 (Milone et al. 2012) have been explained by a range in helium abundances (Norris 2004).

In the cluster NGC $6218(\mathrm{M} \mathrm{12}),[\mathrm{Fe} / \mathrm{H}]=-1.31 \pm 0.028 \mathrm{dex}$, Carretta et al. (2007) found variations in the sodium abundance near the red giant branch (RGB) bump, which the authors believe to be a reflection of different initial helium content in the target stars. The excess of sodium may indicate that a fraction of BL Her variables are helium overabundant. The masses of the BL Her variables are about $0.50-0.60 \mathrm{M}_{\odot}$ (Bono, Caputo \& Santolamazza 1997, with a progenitor mass value of the order of $0.8 \mathrm{M}_{\odot}$ ). In Fig. 9, we present the evolutionary tracks by Chantereau et al. (2016) for the stars with an initial mass of $0.8 \mathrm{M}_{\odot}$ and metallicity $[\mathrm{Fe} / \mathrm{H}]=-1.75$ calculated for different helium abundances ( $\mathrm{Y}=0.248,0.30,0.37,0.425,0.45)$. The figure shows the position of the instability strip, as well as the region occupied by the RR Lyrae variables. As can be seen from the figure, stars with some spread in helium abundance ( $\mathrm{Y}=0.25-0.35)$ can fall into the region of the instability strip, where the BL Her variables are located. This fact may be responsible for the rather small number of the known variables of this type.

As predicted by pulsation models, the pulsation amplitude decreases with increasing helium abundance; and hence, the light curve becomes more sinusoidal in shape while its asymmetry decreases (Marconi et al. 2016). As we assume that Y correlates with the Na excess, then the brightness amplitude and asymmetry should also correlate with the $\mathrm{Na}$ abundance, and that is actually observed (see Fig. 10). This confirms our hypothesis that the helium abundance plays a key role in the properties of the BL Her stars and is crucial for their existence itself.

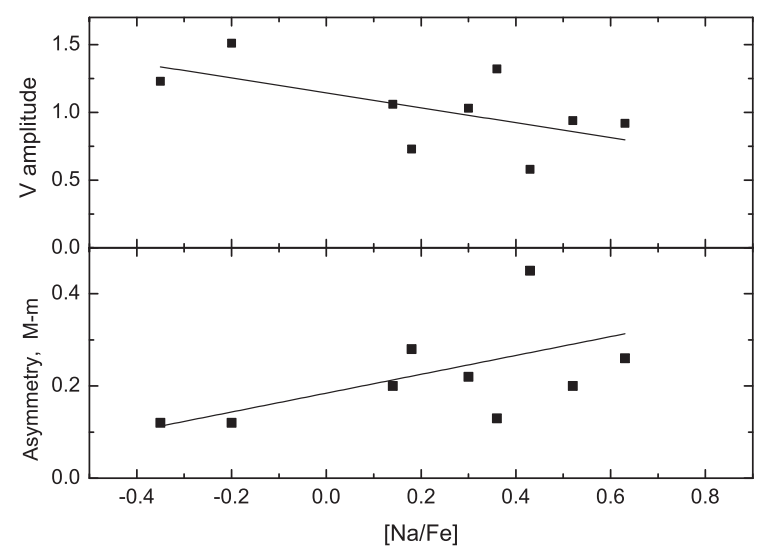

Figure 10. Correlation of the amplitude of brightness variations (top) and asymmetry of the light-curve M-m (bottom figure) with the $\mathrm{Na}$ abundance. The light-curve amplitude decreases with increasing $\mathrm{Na}$ abundance (i.e. helium content), and the curve itself becomes less asymmetrical.

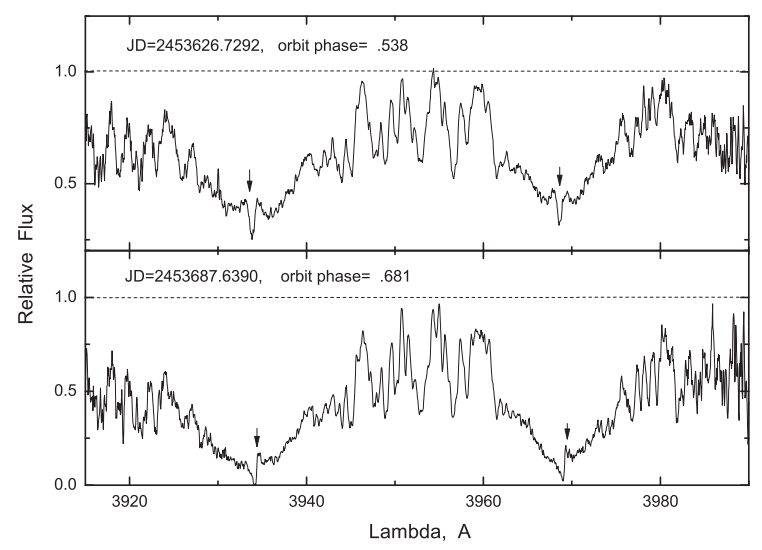

Figure 11. AU Peg. satellite emission lines.

\section{SIGNS OF CIRCUMSTELLAR ENVELOPES}

Two signs of the circumstellar envelope in some of programme stars will be shortly discussed in this section. They are emission lines in the core of Ca II UV lines and dependence of the elemental abundance on condensation temperature. For unusual spectroscopic binary AU Peg, we first discovered the potential emission lines of the companion in the region of $\mathrm{HK} \mathrm{Ca}$ II lines, present in the two studied spectra (see Fig. 11). These lines show the offset in accordance with the orbital movement of the companion. If these are the emission lines of the companion, it cannot be a massive compact object as Harris et al. (1984) proposed before. The existence of a shell is also supported by the presence of the IR excess (McAlary \& Welch 1986) and a P Cygni-like $\mathrm{H} \alpha$ line profile (Vinkó et al. 1998).

Presence of an envelope in AU Peg system is also clearly supported by the plot of abundance versus condensation temperature (Fig. 12). The same dependence is seen for AL Vir (Fig. 13), $\kappa$ Pav (Fig. 14), V1185 Sgr (Fig. 15), and YZ Vir (Fig. 16). It should be noted that all these stars except AU Peg are of the W-Vir-type stars. From these figures, it is seen that volatile elements (like O, S, $\mathrm{Zn}$ ) are more abundant comparing to refractory elements (iron-peak elements, for instance). The former remain in the gaseous shell, and can therefore be accreted by the star, while the latter form dust grain that can be swept out the shell owing to the star radiation. Such a mechanism was considered in connection with abundance anomalies e.g. of $\lambda$ Boo stars (Venn \& Lambert 1990; Charbonneau 1991, 


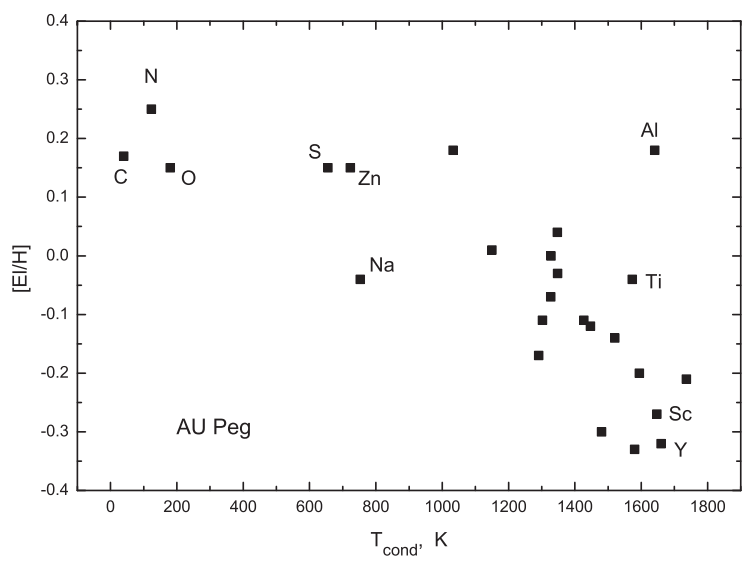

Figure 12. $[\mathrm{El} / \mathrm{H}]$ versus $T_{\text {cond }}$ for $\mathrm{AU}$ Peg.

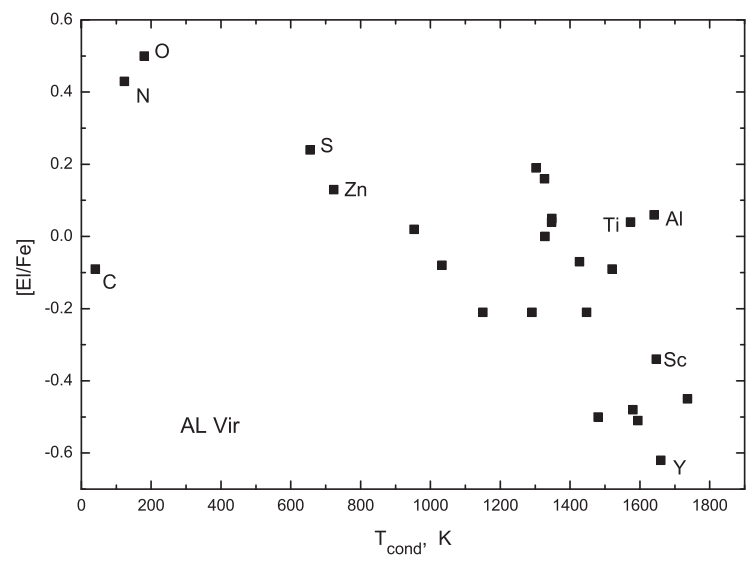

Figure 13. $[\mathrm{El} / \mathrm{H}]$ versus $T_{\text {cond }}$ for AL Vir.

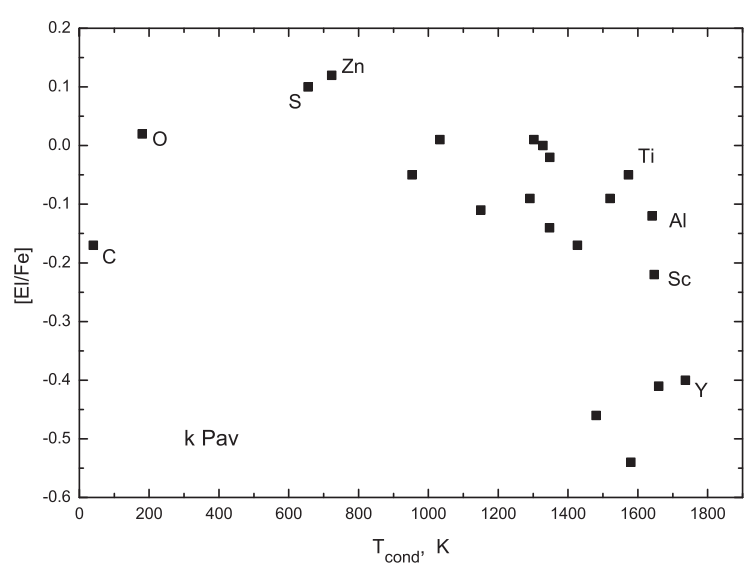

Figure 14. $[\mathrm{E} 1 / \mathrm{H}]$ versus $T_{\text {cond }}$ for $\mathrm{k}$ Pav.

1993; Andrievsky \& Paunzen 2000), W Vir stars (Maas et al. 2007; Lemasle et al. 2015), and RV Tau stars (Giridhar 2000; Giridhar, Lambert \& Gonzalez 2000).

\section{CONCLUSION}

We have spectroscopically investigated sample of Population II variable stars of BL Her, W Vir, and RV Tau types. Abundances of many chemical elements were derived. Among them, nitrogen, oxygen, and sodium were treated under NLTE approximation. These abundances were employed to reveal a possible $\mathrm{Na}-\mathrm{O}$ anticorrelation

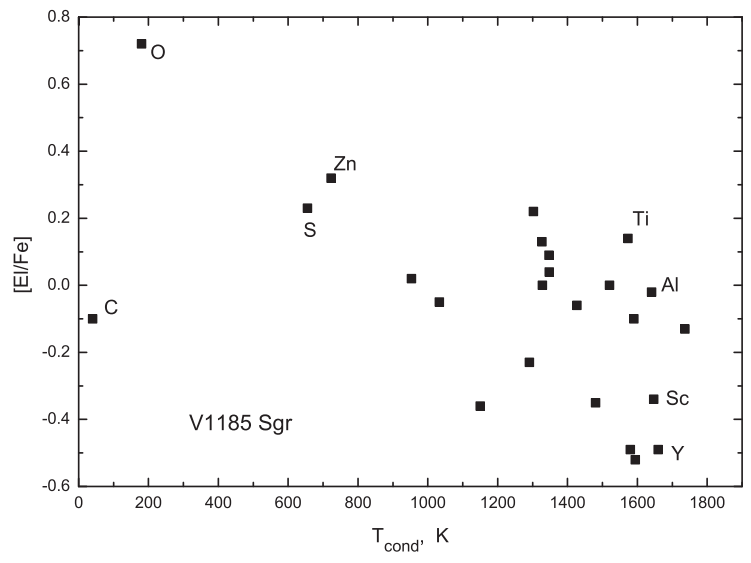

Figure 15. $[\mathrm{El} / \mathrm{H}]$ versus $T_{\text {cond }}$ for V1185 Sgr.

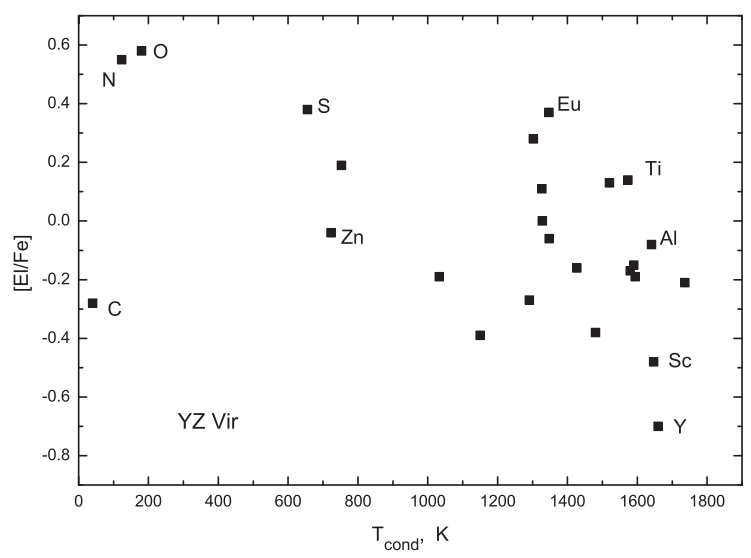

Figure 16. $[\mathrm{El} / \mathrm{H}]$ versus $T_{\text {cond }}$ for $\mathrm{YZ}$ Vir.

that is seen in globular clusters. W Vir and BL Her show oxygen abundance trend with metallicity similar to what is typical for the thick disc and halo field stars. At the same time, a fraction of BL Her stars are apparently sodium rich, while W Vir stars are not. In the plot $\mathrm{Na}-\mathrm{O}$ our limited sample of BL Her stars give a little evidence of the anticorrelation, but being combined with Maas et al. (2007) data on BL Her stars, together they obey $\mathrm{Na}-\mathrm{O}$ relation typical for globular clusters.

We also found an evidence of the shell presence in some programme stars. For unusual BL-Her-type star AU Peg (spectroscopic binary system), we detected for the first time emission in $\mathrm{HK} \mathrm{Ca}$ II lines produced by the companion star.

Finally, we hypothesize that BL-Her-type stars may possess an increased helium abundance. Essentially, only stars with helium overabundance can fall into the region of the instability strip where the BL Her variables are located. This fact may be responsible for the rather small number of the known variables of this type. An origin of their possible overabundance of helium is unknown. One can suppose that some of them may be stars escaped from the bulge of the Galaxy. Nataf et al. (2011) revealed that the metal-rich stellar populations of the Galactic bulge can have $Y \sim 0.35$ based on observational results on the physical properties of the RGB bump.

\section{ACKNOWLEDGEMENTS}

We are grateful to George Wallerstein for valuable discussions and comments and for kindly provided spectra from APO. The authors thank the anonymous referee for her/his very valuable comments 
that significantly improved the paper. I.Y. thanks project ALMACONICYT 31150029 for support. B.L. acknowledges support from Sonderforschungsbereich SFB 881 'The Milky Way System' (subproject A5) of the German Research Foundation (DFG). V.K., S.A., and S.K. acknowledge SCOPES grant no. IZ73Z0-152485 for financial support. V.K. and S.A. kindly acknowledge financial support from the Kenilworth Fund obtained through CRDF.

\section{REFERENCES}

Andrievsky S., Paunzen E., 2000, MNRAS, 313, 547

Andrievsky S. M., Kovtyukh V. V., Wallerstein G., Korotin S. A., Huang W., 2010, PASP, 122, 877

Ballester P., Modigliani A., Boitquin O., Cristiani S., Hanuschik R., Kaufer A., Wolf S., 2000, Messenger, 101, 31

Bensby T., Feltzing S., Lundstroem I., Ilyin I., 2005, A\&A, 433, 185

Bensby T., Feltzing S., Oey M. S., 2014, A\&A, 562A, 71

Bono G., Caputo F., Santolamazza P., 1997, A\&A, 317, 171

Carretta E. et al., 2007, A\&A, 464, 939

Carretta E. et al., 2009, A\&A, 505, 117

Castellani V. et al., 2007, ApJ, 663, 1021

Castelli F., Kurucz R. L., 2004, preprint (arXiv:astro-ph/0405087)

Chantereau W., Charbonnel C., Meynet G., 2016, A\&A, 592, 111

Charbonneau P., 1991, ApJ, 372, L33

Charbonneau P., 1993, in Dworetsky M., Castelli F., Faraggiana R., eds, Proc. IAU Coll. 138, Peculiar versus Normal Phenomena in A-type and Related Stars. Kluwer, Dordrecht, p. 474

Charbonnel C., 2016, in Moreau E., Lebreton Y., Charbonnel C., eds, EAS Publ. Ser. Vol. 80-81, Stellar Clusters: Benchmarks of Stellar Physics and Galactic Evolution - EES2015. p. 177

Chiappini C., 2013, AN, 334, 595

Chiappini C., Frischknecht U., Meynet G., Hirschi R., Barbuy B., Pignatari M., Decressin Th., Maeder A., 2011a, Nature, 472, 454

Chiappini C., Frischknecht U., Meynet G., Hirschi R., Barbuy B., Pignatari M., Decressin Th., Maeder A., 2011b, Nature, 474, 666

Clement Ch. M. et al., 2001, AJ, 122, 2587

Dobrovolskas V. et al., 2014, A\&A, 565A, 121

Drake A. J. et al., 2014, ApJS, 213, 9

Feast M. W., Laney C. D., Kinman Th. D., van Leeuwen F., Whitelock P. A., 2008, MNRAS, 386, 2115

Giridhar S., 2000, IAUS, 177, 117

Giridhar S., Lambert D. L., Gonzalez G., 2000, ApJ, 531, 521

Harris H. C., 1985, AJ, 90, 756

Harris H. C., Wallerstein G., 1984, AJ, 89, 379

Harris H. C., Olszewski E. W., Wallerstein G., 1984, AJ, 89, 119
Kholopov P. N. et al., 1985, Inf. Bull. Var. Stars, 2681, 1

Korotin S. A., Mishenina T. V., 1999, Astr. Rep., 43, 533

Korotin S. A., Andrievsky S. M., Luck R. E., Lepine J. R. D., Maciel W. J., Kovtyukh V. V., 2014, MNRAS, 444, 3301

Kovtyukh V. V., 2007, MNRAS, 378, 617

Kovtyukh V. V., Wallerstein G., Andrievsky S. M., Gillet D., Fokin A. B., Templeton M., Henden A. A., 2011, A\&A, 526, 116

Kupka F., Piskunov N. E., Ryabchikova T. A., Stempels H. C., Weiss W. W., 1999, A\&AS, 138, 119

Kurucz R. L., Furenlid I., Brault J., Testerman L., 1984, Solar Flux Atlas from 296 to $1300 \mathrm{~nm}$

Langer G. E., Hoffman R., Sneden C., 1993, PASP, 105, 301

Lemasle B. et al., 2015, A\&A, 579, A47

Luck R. E., Lambert D. L., 2011, AJ, 142, 136

Lyubimkov L. S., Lambert D. L., Korotin S. A., Poklad D. B., Rachkovskaya T. M., Rostopchin S. I., 2011, MNRAS, 410, 1774

Maas T., Giridhar S., Lambert D. L., 2007, ApJ, 666, 378

Marconi M., Coppola G., Bono G., Braga V., Pietrinferni A., 2016, CoKon, 105,125

McAlary C. W., Welch D. L., 1986, AJ, 91, 1209

Milone A. P., Piotto G., Bedin L. R., Cassisi S., Anderson J., Marino A. F., Pietrinferni A., Aparicio A., 2012, A\&A, 537, 77

Mishenina T. V., Korotin S. A., Klochkova V. G., Panchuk V. E., 2000, A\&A, 353, 978

Nataf D. M., Udalski A., Gould A., Pinsonneault M. H., 2011, ApJ, 730, 118

Norris J. E., 2004, AJ, 612, 25

Pancino E., Britavskiy N., Romano D., Cacciari C., Mucciarelli A., Clementini G., 2015, MNRAS, 447, 2404

Parsons S. B., 1964, ApJ, 140, 853

Reddy B. E., Lambert D. L., Prieto C. A., 2006, MNRAS, 367, 1329

Renzini A. et al., 2015, MNRAS, 454, 4197

Schmidt E. G., Johnston D., Lee K. M., Langan S., 2004, AJ, 128, 2988

Schmidt E. G., Rogalla D., Thacker-Lynn L., 2011, AJ, 141, 53

Soszyński I. et al., 2008, Acta Astron., 58, 293

van Winckel H., 2003, ARA\&A, 41, 391

Venn K. A., Lambert D. L., 1990, ApJ, 363, 234

Vinkó J., Evans N. R., Kiss L. L., Szabados L., 1998, MNRAS, 296, 824

Wallerstein G., 2002, PASP, 114, 689

Wallerstein G., Gonzalez G., 1996, MNRAS, 282, 1236

Wallerstein G., Matt S., Gonzalez G., 2000, MNRAS, 311, 414

Wallerstein G., Gomez Th., Huang W., 2012, Ap\&SS, 341, 89

This paper has been typeset from a $\mathrm{T}_{\mathrm{E}} \mathrm{X} / \mathrm{LT} \mathrm{T} \mathrm{X}$ file prepared by the author. 